\title{
Lipídio: fator de risco e prevenção do câncer de mama
}

\author{
Lipid: risk factor and breast cancer prevention
}

Gabriela Herrmann CIBEIRA ${ }^{1}$

Regina Maria GUARAGNA ${ }^{1}$

\section{R E S U M O}

A hipótese de que uma dieta rica em gordura promova o desenvolvimento do câncer de mama na menopausa é fortalecida por estudos caso-controle, que mostram forte associação positiva entre uma dieta rica em lipídios e as taxas de incidência de câncer de mama. Por outro lado, a ingestão dietética de gordura não parece estar relacionada com o risco de câncer de mama em estudos de coorte. Em vista desses achados conflitantes, tem sido difícil propor qualquer recomendação nutricional para a prevenção do câncer de mama. Estudos com animais e observações recentes em humanos, entretanto, têm mostrado evidências de que a dieta rica em ácido graxo linoléico estimula vários estágios no desenvolvimento de câncer mamário. Alguns estudos ainda mostram que o óleo de peixe, constituído de ácidos graxos $\omega$-3, parece prevenir o câncer pela influência sobre a atividade de enzimas e proteínas relacionadas à proliferação celular. Assim, são necessários estudos epidemiológicos que integrem as interações de ácidos graxos específicos com o catabolismo hormonal, fatores nutricionais protetores e de risco relacionados com o câncer de mama. Nesse trabalho, abordaremos os fatores protetores, de risco e as implicações quali e quantitativas dos ácidos graxos da dieta sobre o câncer de mama.

Termos de indexação: ácidos graxos; dieta; ingestão de gordura; lipídeos; neoplasias mamárias.

\section{A B S T R A C T}

The hypothesis that a high fat diet promotes the development of postmenopausal breast cancer is supported by case-controls studies that show a strong correlation between fat intake and breast cancer rates. On the other hand, high fat intake is not correlated with breast cancer in cohort studies. In view of these conflicting findings, it has been difficult to propose nutritional recommendations for the prevention of breast cancer. Animal studies and recent observations in humans have shown that a diet high in polyunsaturated fatty acids stimulates several stages in the development of mammary cancer. Some evidences show that fish oil-derived $\omega-3$ fatty acids seem to prevent cancer by influencing the activity of enzymes and proteins related to cell proliferation. Thus, epidemiological studies that integrate the interactions of specific fatty acids with hormone catabolism, protective nutritional factors and risk factors associated with breast cancer are needed. In this article we will discuss protective factors, risk factors and qualitative and quantitative implications of dietary fatty acids in breast cancer.

Indexing terms: fatty acids; diet; fat intake; lipids; breast neoplasms.

\footnotetext{
1 Departamento de Bioquímica, ICBS, Universidade Federal do Rio Grande do Sul. Rua Ramiro Barcelos 2600, Anexo, 90035-003, Porto Alegre, RS, Brasil. Correspondência para/Correspondence to: R.M. GUARAGNA. E-mail: <rguaragna@terra.com.br>.
} 


\section{N T R O D U Ç Ã O}

Muitos estudos mostram que durante o último século, a incidência e a mortalidade por câncer de mama variaram muito de um país a outro'. Evidências mostram que a migração de áreas de baixa para locais de elevada incidência da doença contribui para a exposição aos fatores responsáveis pelo aumento de ocorrência do câncer de mama². Tal fato sugere que fatores ambientais e relacionados ao estilo de vida são importantes no desenvolvimento da neoplasia ${ }^{3}$. Há evidências de que o efeito da gordura da dieta seja exercido depois do início da tumorgênese e que esse processo geralmente aumenta com o conteúdo de gordura ${ }^{4}$. Freedman et al. ${ }^{5}$ concluíram que a ingestão de gordura aumenta a tumorgênese mamária em ratos, independentemente da elevada ingestão energética. Devido ao livre acesso ao alimento, a incidência de tumores mamários nestes animais aumentou com a elevada quantidade de gordura ingerida, tendo o conteúdo de ácido linoléico contribuído fortemente para o efeito da gordura sobre a tumorgênese.

Alguns estudos associam o vegetarianismo (elevada ingestão de vegetais, frutas, cereais, fibras, gordura insaturada e baixo consumo de gordura saturada) com baixos níveis sanguíneos de estrógenos e excreção urinária reduzida do hormônio, aparentemente devido à excreção fecal ${ }^{6}$. Há evidências sugerindo esse estilo de vida como um fator protetor contra o câncer de mama ${ }^{7}$, apontando os micronutrientes ${ }^{8}$ e as fibras ${ }^{9}$ como protetores contra a neoplasia, porém, nem todos os resultados estão de acordo com essa afirmação ${ }^{10}$. Silva et al $^{7}$. indicaram que a redução na ingestão de carne vermelha poderia diminuir o risco de câncer de mama, contribuindo ainda mais para o fator protetor do vegetarianismo ${ }^{11}$. Além disso, mulheres que consomem grandes quantidades de carne vermelha estão expostas a agentes mutagênicos e cancerígenos. Esse alimento cozido em altas temperaturas contém aminas heterocíclicas, que são mutagênicas para o câncer de mama ${ }^{12}$.
De Stefani et al. ${ }^{13}$ avaliaram os efeitos da ingestão de carne sobre o risco de neoplasia provocada pela exposição a aminas heterocíclicas. Foram analisados 352 pacientes com a doença e 382 indivíduos controles. O estudo revelou correlação da doença com a exposição a aminas heterocíclicas. O risco relativo do quartil mais alto foi de 3,34 (95\% intervalo de confiança 1,85-6,02), demonstrando que a ingestão de carne vermelha e os produtos químicos formados a partir do cozimento do alimento parecem ser um forte fator de risco na carcinogênese mamária em humanos.

Apesar dos vários estudos que têm procurado associar a gordura da dieta e o câncer de mama, nenhuma conclusão pode ser ainda firmada, pois se trata de uma doença multifatorial cujos processos ainda não são totalmente esclarecidos. Este trabalho tem por objetivo apresentar possíveis mecanismos por meio dos quais os lipídios possam atuar como fatores de risco ou protetores do câncer de mama.

\section{Epidemiologia}

As taxas de incidência de câncer de mama variam aproximadamente cinco vezes ${ }^{14}$ entre os continentes e apresentam forte correlação com a disponibilidade per capita nacional de gordura da dieta ${ }^{2,7}$. Essa associação parece ser devido ao uso de gorduras saturadas, ao invés de gorduras polinsaturadas ${ }^{15}$. No entanto, o uso de dados per capita para caracterizar a ingestão dietética é questionável e a qualidade dos registros nacionais de incidência e mortalidade pela neoplasia pode ser diferente em relação a outros países. Por outro lado, comparações entre populações distintas possibilitam associações entre os hábitos alimentares e o câncer de mama.

Na Indonésia, o consumo de gordura é relativamente baixo (15\% - 20\% da energia total) e a neoplasia mamária é a segunda malignância mais comum entre as mulheres. Um estudo caso controle, realizado no país, mostrou forte e consistente associação entre o consumo de gordura na dieta e o risco de câncer de mama ${ }^{16}$. O risco 
relativo do quartil mais alto em relação ao quartil mais baixo para a gordura consumida foi de 8,47 (95\% intervalo de confiança 4,03-17,8). Esse estudo, entretanto, não levou em consideração o tipo de gordura ingerida, mostrando que são necessários estudos mais detalhados que relacionem ácidos graxos específicos com a ocorrência de câncer de mama.

Mulheres chinesas consumindo aproximadamente $25 \%$ de energia proveniente de gordura possuem taxas de câncer de mama menores que mulheres americanas com ingestão total de gordura semelhante ${ }^{2}$. Essa observação revela que o tipo de gordura ingerido pode exercer um papel fundamental no risco do desenvolvimento do câncer mamário, como exemplificado por estudos que associam as baixas taxas de incidência da doença nos países mediterrâneos à gordura monoinsaturada consumida ${ }^{17}$.

Embora a idade média da menarca nos países ocidentais seja hoje de 12 - 13 anos de idade, na China rural a idade típica tem sido aproximadamente 17 - 18 anos, similar ao observado nos países ocidentais há 200 anos $^{18}$. Comparado com meninas, que tiveram a menarca aos 16 anos, aquelas que a apresentaram 2 a 5 anos mais precocemente, revelaram maiores riscos de desenvolverem câncer de mama, de 10\% a $30 \%$. É possível que a dieta consumida durante a infância e adolescência exerça um papel importante no risco futuro de adoecimento, já que a neoplasia de mama tem um longo período de latência, e a puberdade é caracterizada por intensas mitoses e crescimento tecidual com aumento na altura, peso e pleno desenvolvimento ${ }^{20}$. Uma observação semelhante em relação à idade da menopausa foi abordada por Vogel ${ }^{19}$ em sua revisão. Considerando-se mulheres que entraram na menopausa entre os 45 e 55 anos como grupo de referência (a média de idade da menopausa nos EUA é aproximadamente 51 anos), mulheres que tiveram a menopausa aos 55 anos ou mais, possuíram 50\% mais chances de desenvolverem, subseqüentemente, o câncer de mama, e mulheres que cessaram a menstruação aos 45 anos ou mais jovens tiveram $30 \%$ menos chances de desenvolvê-lo ${ }^{19}$. A explicação provável para essas observações seria o elevado nível de estrógenos circulantes, aos quais a mulher é exposta entre a menarca e a menopausa. Dessa forma, parece que uma dieta rica em gordura poderia afetar a biodisponibilidade do hormônio pela elevação na concentração plasmática de ácidos graxos livres ${ }^{21}$.

Apesar de ser considerado como um câncer de relativamente bom prognóstico, se diagnosticado e tratado precocemente, as taxas de mortalidade por câncer de mama continuam elevadas no Brasil. No País, mortes causadas pela neoplasia vêm aumentando significativamente nos últimos vinte anos, acentuando-se a partir da década de 90. Essa taxa, entre 1979 e 2000, passou de 5,77/100.000 para 9,74/100.000, correspondendo a uma variação percentual relativa de $+80,73 \%$. Em 2003, o número de mortes pela neoplasia mamária foi de 9335 e 41610 casos novos foram observados. Esses números correspondem a taxas brutas de mortalidade e incidência de 10,40/100.000 e 46,35/100.000, respectivamente 22 .

Uma pesquisa realizada no estado de São Paulo ${ }^{23}$ estudou o consumo alimentar de mulheres no climatério e constatou que apenas 15\% da amostra ( $n=154)$ apresentou consumo adequado de gordura. Mondini et al. ${ }^{24}$ estudaram as modificações no padrão da alimentação urbana brasileira entre 1962 e 1988. As fontes de dados para o estudo foram duas pesquisas nacionais de orçamentos familiares, realizadas no início da década de 60 (1961-1963), e no final da década de 80 (1987-1988) e um inquérito nacional sobre consumo alimentar, realizado em meados da década de 70 (1974-1975), restringindo-se a análise a sete áreas metropolitanas estudadas em comum pelas três pesquisas. O estudo evidenciou uma diminuição no consumo de ácidos graxos saturados e um aumento na ingestão de ácidos graxos poliinsaturados. Esse fato se deu em virtude da substituição do consumo de banha de porco e toucinho por óleos vegetais, em particular o óleo de soja. 
A saúde de uma população é o resultado de interações entre fatores genéticos e ambientais. Atualmente as sociedades industrializadas são caracterizadas pelo elevado consumo de gordura saturada e ácidos graxos trans, baixo consumo de ácidos graxos ômega 3, carboidratos complexos e fibras alimentares. Além disso, a relação $\omega-6 / \omega-3$ aumentou oito vezes durante a nossa evolução, se compararmos com o consumo de nossos ancestrais durante o período Paleolítico. As mudanças na nossa dieta, principalmente nos últimos 100 anos, são potentes promotores de várias doenças, entre elas, o câncer de mama. Além da dieta, hábitos sedentários de estilo de vida e a exposição a substâncias tóxicas interagem com processos bioquímicos, geneticamente controlados, contribuindo ainda mais para elevar a incidência da doença ${ }^{25}$.

\section{Qualidade do lipídio dietético}

Diferentes tipos de gordura, como ácidos graxos saturados, insaturados, poliinsaturados e trans, têm sido relacionados com o câncer de mama ${ }^{26}$. Alguns estudos ${ }^{4,21}$ mostram que vários passos do processo tumorgênico, incluindo iniciação, promoção, latência, crescimento e metástases, podem ser influenciados pela gordura da dieta. O ácido linoléico (altamente consumido nos países ocidentais) tem sido considerado como a base na indução e nas metástases de tumores ${ }^{21}$, sendo que a relação ômega $6(\omega-6)$ : ômega 3 $(\omega-3)$ parece ser fundamental, já que os ácidos graxos $\omega$-3 (ácido linolênico) são inibidores competitivos dos efeitos dos ácidos graxos $\omega-6$.

Matsuba et al. ${ }^{27}$ examinaram os efeitos de três óleos sobre a tumorgênese animal, pois se diferenciavam nas concentrações de ácido linoléico e ácido linolênico (taxas de $\omega$-6: $\omega$-3). Comparado com o óleo de girassol (70,0\% ácido linoléico e 0,1\% ácido linolênico) e com o óleo de soja (50,0\% ácido linoléico e 5,0\% ácido linolênico), o óleo de perila (15,0\% ácido linoléico e 65,0\% ácido linolênico), usado principalmente no Japão, preveniu tumores de mama em ratos.
Contudo, a ingestão de ácido linoléico tem aumentado nas duas últimas décadas ${ }^{26}$. Dietas contendo óleo de milho (rico em ácido linoléico) parecem aumentar a tumorgênese em ratos, embora o óleo de peixe (rico em ácido eicosapentaenóico e ácido docosahexaenóico) reduza a carcinogênese ${ }^{21}$.

Holmes et al. ${ }^{28}$ e Sugano et al. ${ }^{29}$ têm mostrado o ácido linolênico ( $\omega$-3) como sendo elemento protetor no risco de câncer de mama. A proteção do $\omega$-3 sobre este câncer pode estar associada aos seus metabólicos, que são os eicosanóides com ação pró-inflamatória menos potentes. Além disso, este efeito parece ser devido à influência do $\omega$-3 na atividade de enzimas e proteínas relacionadas à sinalização intracelular e à proliferação celular²1.

Folsom et al..$^{30}$ avaliaram o efeito do ácido linolênico sobre o câncer de mama a partir da ingestão de peixe de 41836 mulheres entre 55 e 69 anos. A coleta de dados se deu por meio de um questionário de freqüência alimentar, entretanto não foi observada associação protetora entre o lipídio e a neoplasia. Deve-se levar em consideração a variabilidade na composição e no teor de $\omega$-3 depositado no peixe, pois depende do plâncton do qual se alimenta, relacionado com o clima e a sazonalidade. Embora alguns óleos sejam naturalmente ricos neste ácido graxo, o processo industrial de hidrogenação parcial diminui a quantidade desse lipídio no produto ${ }^{31}$.

Os japoneses consomem, em média, 26\% da energia proveniente de gordura com uma razão de lipídio poliinsaturado para saturado de 1-2:1 e de ácidos graxos $\omega$ - 6 para $\omega$-3 de 4:1 (nos países ocidentais essa razão chega a 10:1). Essa elevada ingestão de $\omega$-3 pelos japoneses, não é proveniente apenas de peixes e frutos do mar, mas também de óleos vegetais, como o óleo de soja. Estudos comparativos entre japoneses vivendo no Japão, com aqueles vivendo nos Estados Unidos e americanos brancos, mostraram que as concentrações de $\omega-3$ em japoneses morando no Japão foram três vezes maiores que aqueles morando nos Estados Unidos. É interes- 
sante que a proporção de $\omega$ - 6 é maior em americanos que em japoneses, refletindo a diferença nas taxas de $\omega-6$ para $\omega-3$ na dieta. Similarmente, o leite de mães japonesas contém teores mais elevados de $\omega$-3 que o das mães ocidentais ${ }^{29}$. Os ácidos graxos $\omega-3$ mais consumidos nos Estados Unidos são o ácido linolênico (ALA), o ácido eicosapentaenóico (EPA) e o ácido docosahexaenóico (DHA). A relação $\omega-6$ para $\omega$-3 neste país é de 9:1 e, mesmo tendo havido um ligeiro decréscimo dessa proporção desde 1985, essa relação ainda permanece bem mais alta que a recomendada $(2-3: 1)^{32}$.

Algumas evidências sugerem que o óleo de oliva e outras fontes de ácidos graxos monoinsaturados podem, modestamente, diminuir o risco de câncer de mama ${ }^{11}$. Um estudo multicêntrico mostrou uma relação mais forte entre o ácido oléico e o câncer de mama que qualquer outro monoinsaturado, sugerindo que o consumo de óleo de oliva pode proteger contra o câncer de mama ${ }^{33}$. Na Itália e na Espanha, onde o óleo de oliva é a principal fonte de ácido oléico da dieta, a incidência de câncer de mama é menor que na América do Norte e nordeste da Europa. Essa proteção pode ser atribuída à elevada quantidade de antioxidantes presentes no óleo. O óleo de oliva (rico em $\omega$-9 ácidos graxos monoinsaturados, mas baixo em $(\omega-6)$ é composto, principalmente, por vários agentes quimiopreventivos, como tocoferóis, carotenóides, esqualeno, polifenóis, ligninas e outros quimioprotetores naturais ${ }^{21}$. Esses componentes podem proteger contra o dano oxidativo, aumentando a reparação de processos e aumentando a resistência à peroxidação ${ }^{33}$.

Entre mulheres gregas que consomem $42 \%$ da sua energia proveniente de gordura, principalmente do óleo de oliva, as taxas de câncer de mama são significativamente menores que as das mulheres nos Estados Unidos, cuja ingestão energética proveniente da gordura é de aproximadamente $35 \%{ }^{21}$. O câncer e as doenças cardiovasculares causam proporcionalmente três vezes mais mortes nos Estados Unidos que em Creta. A dieta grega se caracteriza pela elevada ingestão de antioxidantes, elevada ingestão de óleo de oliva, baixo consumo de gordura saturada e uma taxa de ácidos graxos $\omega$-6 para $\omega$-3 de aproximadamente, 2:1, ao invés de 15:1, como na dieta dos países ocidentais e do norte da Europa ${ }^{17}$

Mais de um quarto do total de calorias ingerido é normalmente fornecido por ácidos graxos que contêm pelo menos uma dupla ligação. A configuração usual dessas ligações duplas é a configuração cis. Os ácidos graxos trans (AGTs) são formados em processos tecnológicos (hidrogenação industrial) e microbiológicos (no estômago de ruminantes) por isomerização de dupla ligação cis para dupla ligação trans. Industrialmente o processo de transformação de óleos, que são líquidos, à temperatura ambiente para gorduras de aspecto sólido, a essa temperatura, realiza-se por meio da hidrogenação catalítica de duplas ligações. Esse processo aumenta a ocorrência de ácidos graxos com ponto de fusão acima da temperatura ambiente e produz os chamados ácidos graxos (insaturados) trans em grande quantidade. Dentre os ácidos graxos trans obtidos pelo processo industrial de hidrogenação parcial ou total de óleos vegetais ou marinhos o mais comum é o ácido eláidico (C18:1, t9), isômero trans do ácido oléico, ambos ácido graxo monoinsaturado. O ácido eláidico é o principal AGTs da dieta e contribui em, aproximadamente, $80 \%$ - 90\% da quantidade total de AGTs nos alimentos ${ }^{34}$. Os ácidos graxos trans são encontrados em gorduras vegetais hidrogenadas, margarinas sólidas ou cremosas, cremes vegetais e, conseqüentemente, nos biscoitos, sorvetes, pães, batatas fritas, pastelarias, bolos, tortas, massas, entre outros alimentos.

Estudos de Mensink et al. ${ }^{35}$ demonstraram que substituindo a dieta rica em ácido oléico por uma dieta rica em ácido eláidico, o colesterol total aumenta, enquanto a HDL diminui. A função das membranas celulares depende da configuração tridimensional de seus ácidos graxos. Como os ácidos graxos trans assumem configuração semelhante aos ácidos graxos saturados, se agrupam e se compactam firmemente. A ingestão 
destes pode influenciar na fluidez de membrana, prejudicando a função celular. E, por este motivo, eles têm sido associados ao risco de câncer de mama $^{36}$.

França, Canadá, Inglaterra, Dinamarca, Nova Zelândia e outros países têm recomendado valores de consumo de $2 \%$ a $5 \%$ de gordura trans em relação à ingestão total de energia diária ${ }^{37}$. No Brasil, Bortoloto et al. ${ }^{38}$ determinaram a quantidade de ácido graxo trans em diversas margarinas e sorvetes nacionais. Os resultados, alarmantes, detectaram 38\% e $40 \%$ de AGT em relação aos lipídios totais em algumas margarinas e sorvetes, respectivamente. Entretanto, a quantificação de AGTs ingeridos com alguma precisão é muito difícil, mesmo usando questionário específico. No Brasil, desde 1997, as gorduras trans deixaram de ser computadas como insaturadas, sendo incluídas no total de saturadas e não separadamente ${ }^{39}$. Desta forma, para avaliar o risco da nossa população às doenças que estão associadas, em parte, a este lipídio, estão sendo realizadas análises quantitativas de AGT totais, armazenados no tecido adiposo (mamário, retroperitonial, subcutâneo e visceral), tanto em indivíduos com índice de massa corporal maior que $40 \mathrm{~kg} / \mathrm{m}^{2}$ e menor que $30 \mathrm{~kg} / \mathrm{m}^{2}$. O depósito do AGT é maior no tecido adiposo visceral (8,7\%), se comparado a outros tecidos como o subcutâneo $(6,9 \%)$ e retroperitonial (6,3\%). Na mama, entretanto, o depósito do ácido graxo foi de 4,7\%. Este fato pode estar relacionado com o consumo de alimentos com elevados teores de gorduras hidrogenadas e baixo consumo de lipídios essenciais ${ }^{40}$, principalmente os ácidos $\omega$-3.

$\mathrm{O}$ ácido linoléico conjugado (CLA), um grupo de isômeros posicionais e geométricos do ácido linoléico, tem mostrado ser um potente anticarcinógeno em tumores de pele, estômago e mama ${ }^{41}$. Alguns de seus isômeros são seletivamente ativos prevenindo câncer, favorecendo perda de peso, reduzindo a quantidade de gordura em tecido adiposo, atenuando as taxas do colesterol indesejável (LDL), alterando favoravelmente o sistema imune, reduzindo inflamações nas articulações, combatendo a formação de placas de ateroma, entre outras propriedades ${ }^{42}$. O leite e outros laticínios são boas fontes de CLA, devido à biohidrogenação de ácidos graxos insaturados da dieta pelas bactérias do rúmen ${ }^{43}$. É interessante observar que a alimentação da vaca em muito influencia o teor de CLA no leite. O leite de animais alimentados exclusivamente com pasto contém cerca de $400 \%$ mais CLA que o daqueles alimentados com grãos e silagem ${ }^{43}$. Alguns estudos ${ }^{41}$ demonstram ser o CLA um agente redutor da incidência de diversos tipos de câncer. Sempre se adverte que a variante ativa contra o câncer é o isômero cis-9, trans-11. Na gordura típica do leite, este é o principal isômero presente, variando de $80 \%$ a $90 \%$ do CLA total. É interessante ressaltar que o ácido linoléico, em altas concentrações, tende a aumentar a ocorrência de câncer, e que somente o isômero cis-9, trans-11 atua como anticarcinogênico natural. Outro isômero do ácido linoléico (isômero trans-10, cis-12) também exerce controle sobre a estocagem de gordura como tecido adiposo, pois provoca diminuição do apetite, desencadeando aumento do metabolismo e, conseqüentemente, perda de gordura corporal. Em 1998, West et al. ${ }^{44}$, ao estudarem o efeito do CLA na dieta de ratos de laboratórios, observaram que o CLA trans-10, cis-12 proporcionou diminuição de $43 \%$ a $88 \%$ do tecido adiposo dessas cobaias, sendo que a região abdominal foi a mais sensível à ação do CLA.

\section{Câncer de mama, estrógeno e ingestão de gordura}

Uma dieta rica em gordura aumenta os níveis séricos de estrógeno ${ }^{45}$. Por outro lado, uma dieta com baixo teor de gordura pode diminuir os níveis de estrógeno circulante. Esses têm sido relacionados com o câncer de mama, principalmente devido às suas ações fisiológicas estimulatórias nas glândulas mamárias. Elevados níveis de estrógeno e progesterona, juntamente com baixos níveis de gonadotrofinas, caracterizam a menopausa. Nessa fase, esse hormônio não é mais 
secretado pelos ovários, mas sim produzido por meio da conversão periférica de precursores andrógenos ${ }^{46}$. Os estrógenos são formados pela aromatização de precursores androgênicos circulantes numa variedade de tecidos incluindo músculo, tecido adiposo, folículo capilar, fígado, hipotálamo, entre outros. Tal aromatização periférica é uma importante fonte de estrógenos responsáveis por $40 \%$ de toda a formação do estrógeno em mulheres na puberdade e 100\% em mulheres na menopausa. A taxa de formação de estrógeno e de andrógenos circulantes é aumentada com a obesidade ${ }^{47}$. Dessa forma, uma dieta rica em gordura poderia afetar a biodisponibilidade do hormônio pela elevação da concentração de ácidos graxos séricos livres ${ }^{21}$. Mannisto et al. ${ }^{48}$ sugeriram que o café e o $\beta$-caroteno podem atuar como fatores protetores do câncer de mama, por diminuírem os níveis de estrógeno biodisponíveis no sangue e serem potentes antioxidantes, respectivamente. Mostraram ainda que o elevado consumo de leite parece estar associado com o aumento do risco de câncer de mama Entretanto, mulheres que estavam sendo submetidas ao tratamento do câncer de mama subestimaram seu consumo de leite. Tal fato parece justificar as falsas associações de que apenas o consumo total de leite permanece como fator de risco para a doença (odds ratio $=2.2$, $95 \%$ intervalo confiança $=1,0-4,9)$. Por outro lado, o consumo de carne de ave foi encontrado como sendo fator protetor para o câncer de mama e independente de vieses (odds ratio $=0,4,95 \%$ intervalo confiança 0,2-0,9).

Embora algumas pesquisas realizadas com mulheres não tenham fornecido resultados consistentes entre o consumo de gordura e o desenvolvimento do câncer mamário ${ }^{26}$, estudos em ratos têm revelado que o elevado consumo de gordura, principalmente ácidos graxos derivados do ácido linoléico (cis, cis-C 18:2 $\omega$-6), aumenta o risco de desenvolvimento de tumores mamários ${ }^{4}$. A exposição a elevados níveis maternos de estrógenos circulantes durante a gravidez poderia exercer um papel importante no risco de câncer de mama entre as filhas ${ }^{46}$. O tamanho do recém nascido está correlacionado com o ganho de peso materno e com a elevada ingestão de ácidos graxos poliinsaturados (PUFAs) da mãe, refletindo nos níveis de exposição do feto ao estrógeno ${ }^{46}$.

Hilakivi-Clarke et al. ${ }^{49}$ hipotetizaram que alimentando ratas grávidas com uma dieta rica em gordura, os níveis circulantes de estradiol aumentariam e o risco de desenvolvimento de tumores mamários entre as proles dessas fêmeas também. No estudo, um grupo de ratas grávidas foi alimentado com uma dieta contendo entre 12\% e 16\% (baixa ingestão $\omega$-6) das calorias provenientes do óleo de milho, que contém principalmente $\omega-6$, e outro com uma dieta contendo entre $43 \%$ e $46 \%$ dessa gordura (alta ingestão $\omega-6$ ). Os resultados obtidos mostraram que as concentrações plasmáticas de estradiol foram significativamente maiores entre as fêmeas grávidas alimentadas com a dieta rica em $\omega-6$ e que a prole dessas ratas apresentou incidência maior de tumores mamários.

\section{I S C U S S Ã O}

A possível associação entre a gordura da dieta e o risco de câncer de mama após a menopausa tem sido foco de vários estudos ginecológicos $^{50}$, de revisão ${ }^{2}$, de coorte ${ }^{51}$ e caso-controle, mas a natureza dessa associação ainda parece controversa. Numa análise de seis estudos prospectivos ${ }^{14}$ com mais de 200 casos de câncer de mama, não foram encontradas evidências positivas entre a ingestão total de gordura e o risco de câncer de mama. Além disso, foi observado, ainda, nesse estudo que um grupo de mulheres, consumindo quantidades pequenas de gordura (menos que 15\% da energia total), apresentou duas vezes maior risco de desenvolver câncer de mama. Comparando o quintil com maior ingestão de gordura com o quintil de menor ingestão, o risco relativo multivariado acumulado de câncer de mama foi de 1,05 (intervalo confiança 95\% 0,94-1,16). Para comparação dos 
valores do mais alto quintil com os do mais baixo, os resultados dos testes para heterogeneicidade entre os estudos analisados não indicaram uma diferença significativa para a ingestão qualquer nutriente.

Têm sido postulados que a elevada ingestão de gordura e de ácidos graxos específicos, incluindo ácidos graxos saturados, ácido linoléico e ácidos graxos insaturados trans, aumenta o risco de câncer de mama. Porém, um estudo conduzido por Holmes et al. ${ }^{28}$ não encontrou evidências de que a redução na ingestão de gordura total ou dos ácidos graxos citados acima estivesse associada com a diminuição do risco de câncer de mama. Das 88795 mulheres que não possuíam a doença no início do estudo, foram constatados 2956 casos da neoplasia depois de 14 anos de acompanhamento. Comparado com mulheres que tinham entre $30,1 \%$ e $35 \%$ da energia proveniente de gordura, mulheres que consumiram $20 \%$ ou menos tiveram um risco relativo para câncer de mama de 1,15 (95\% intervalo de confiança, 0,73-1,80). Do mesmo modo, Graham et al. ${ }^{52}$ não observaram associação significativa do risco desse câncer com algum tipo específico de gordura. Foi observado que a baixa ingestão de lipídios ou algum tipo de ácido graxo específico não está associada com a diminuição do risco de câncer de mama. Jakovljevic et al. ${ }^{26}$ acompanharam 2956 mulheres com diagnóstico de câncer mamário durante 14 anos. Foram identificadas fracas associações inversas entre a gordura monoinsaturada e a neoplasia de mama. Foi constatada uma leve associação positiva entre o $\omega$-3 e a doença. De qualquer forma, não foram observadas associações de elevada magnitude entre a menor ingestão total de gordura ou tipos específicos do alimento com a diminuição no risco de câncer de mama.

Por outro lado, Howe et al. ${ }^{53}$ conduziram um estudo com 56837 mulheres e demonstraram associação positiva entre câncer de mama e ingestão total de gordura com um risco relativo de $1,35(p=0,52)$. Kushi et al. ${ }^{54}$ encontraram relação positiva entre a ingestão de gordura e o risco de desenvolverem a doença, sendo a ingestão de ácido linoléico também associada com esta neoplasia. Um estudo realizado no Canadá, por Howe et al. ${ }^{55}$, analisou 4312 casos de câncer de mama e 5978 controles. O risco relativo foi de $1,35(p<0,0001)$ para um aumento de $100 \mathrm{~g}$ na ingestão diária de gordura, embora o risco tenha sido mais forte em mulheres pós-menopausa (risco relativo $=1.48 ; p<0,001)$. A magnitude desse risco relativo poderia, entretanto, estar sendo distorcida, uma vez que a mudança de $100 \mathrm{~g}$ na ingestão de gordura é impossível para a grande maioria das mulheres, já que a média de ingestão tende a ser $70 \mathrm{~g}$ por dia.

Frente aos conhecimentos científicos atuais, a Sociedade Americana de Câncer (ACS) preconiza, para a sua prevenção, a manutenção de níveis adequados de peso corporal; recomenda também limitar o consumo de carnes vermelhas, especialmente as processadas e ricas em gordura, e preferir grãos integrais a grãos refinados e açúcares ${ }^{56}$.

\section{CONCLUSÃO}

O papel específico dos fatores dietéticos na causa do câncer de mama não se encontra completamente esclarecido. O padrão da dieta ocidental tem se modificado com o passar do tempo, tornando-se mais rica em gordura e menos saudável, contribuindo, provavelmente, para o aumento nas taxas de incidência de câncer de mama. O processo de industrialização dos países favorece a essa mudança, à medida que incentiva o consumo de fast foods e alimentos processados, cujo teor de gordura trans é bastante elevado. Por outro lado, o consumo de $\omega-3$ nos países orientais permanece alto e é visto como fator protetor contra o risco da doença, a medida que a relação $\omega-6 / \omega$-3 é de, aproximadamente, 1-2:1.

A suposição de que o consumo de gordura seja um dos mais importantes fatores de risco para o câncer de mama tem tido importantes implicações para os guias dietéticos, e isso tem sido a primeira justificativa para as recomendações de redução na ingestão total do nutriente. 
Fica claro que a qualidade da dieta e o estilo de vida contribuem para o desenvolvimento de neoplasias como o câncer de mama, visto que diferentes tipos de ácidos graxos desempenham papéis distintos em relação a essa doença. Em função dos dados controversos encontrados em diversos estudos, tornam-se necessárias observações mais longas e com maior número de indivíduos. E somente a partir desses estudos, será possível determinar a relação exata entre o hábito cultural e o padrão de dieta com o risco de desenvolvimento de câncer de mama.

\section{REFERÊ NCIAS}

1. Willett WC. Specific fatty acids and risks of breast and prostate cancer: dietary intake. Am J Clin Nutr. 1997; 66(6 Suppl):1557s-63s.

2. Willett WC. Fat, energy and breast cancer. J Nutr. 1997; 127(5 Suppl):912s-3s.

3. Willett WC. Diet and cancer: one view at the start of the millennium. Cancer Epidemiol Biomarkers Prev. 2001; 10(1):3-8.

4. Rogers $A E$. Diet and breast cancer: studies in laboratory animals. J Nutr. 1997; 127(5 Suppl): 933s-5s.

5. Freedman LS, Clifford CK, Messina M. Meta-analysis of animal experiments: elucidating relationships between dietary fat and mammary tumor development in rodents. Adv Exp Med Biol. 1994; 364:93-100.

6. Willett WC. Dietary fat intake and cancer risk: a controversial and instructive story. Semin Cancer Biol. 1998; 8(4):245-53.

7. Silva IDS, Mangtani $P, M c C o r m a c k ~ V$, Bhakta $D$, Sevak L, McMichael AJ. Lifelong vegetarianism and risk of breast cancer: a population-based case-control study among South Asian migrant women living in England. Int J Cancer. 2002; 99(2):238-44.

8. Lipkin $M$, Newmark HL. Vitamin $D$, calcium and prevention of breast cancer: a review. J Am Coll Nutr. 1999; 18(5 Suppl):392s-7s.

9. Willett WC. Diet and cancer. Oncologist. 2000; 5(5):393-404.

10. Key TJ, Fraser GE, Thorogood M, Appleby PN, Beral $V$, Reeves $G$, et al. Mortality in vegetarians and nonvegetarians: detailed findings from a collaborative analysis of 5 prospective studies. Am J Clin Nutr. 1999; 70(3 Suppl):516s-24s.
11. Kushi L, Giovannucci E. Dietary fat and cancer. Am J Med. 2002; 113(Suppl 9B):63s-70s.

12. Zheng W, Gustafson DR, Sinha R, Cerhan JR, Moore $D$, Hong $C P$, et al. Well-Done meat intake and the risk of breast cancer. J Natl Cancer Inst. 1998; 90(22):1724-9.

13. De Stefani E, Ronco A, Mendilabarsu M, Guidobono M, Denco-Pellegrini H. Meat intake, heterocyclic amines, and risk of breast cancer: a case control study in Uruguay. Cancer Epidemiol Biomarkers Prev. 1997; 6(8):573-81.

14. Hunter DJ, Spiegelman D, Adami HO, Buson L, Van den Brandt PA, Folsom AR, Fraser GE, et al. Cohort studies of fat intake and the risk of breast cancer - a pooled analysis. New Engl J Med 1996 Feb 8; 334(6): 356-61.

15. Zock PL, Katan MB. Linoleic acid intake and cancer risk: a review and meta-analysis. Am J Clin Nutr. 1998; 68(1): 142-53.

16. Wakai K, Dillon DS, Ohno Y, Prihartono J, Budiningsih S, Ramli M, et al. Fat intake and breast cancer risk in an area where fat intake is low: a case-control study in Indonesia. Int J Epidemiol. 2000; 29(1):20-8.

17. Simopoulos AP. The Mediterranean diets: what is so special about the diet of Greece? The scientific evidence. J Nutr. 2001; 131(11 Suppl):3065s-73s.

18. Wyshak G, Frisch RE. Evidence for a secular trend in age at menarche. N Engl J Med. 1982; 306(17):1033-5.

19. Vogel VG. Breast cancer prevention: a review of current evidence. CA Cancer J Clin. 2000; 50(3):156-70.

20. Frazier AL, Li L, Cho E, Willett WC, Colditz GA. Adolescent diet and risk of breast cancer. Cancer Causes Control. 2004; 15(1):73-82.

21. Bartsch H, Nair, J, Owen RW. Dietary polyunsaturated fatty acids and cancers of the breast and colorectum: emerging evidence for their role as risk modifiers. Carcinogenesis. 1999; 20(12):2209-18.

22. Brasil. Ministério da Saúde. Instituto Nacional do Câncer. Estimativas da incidência e mortalidade por câncer. Rio de Janeiro: INCA; 2003.

23. Montilla RNG, Marucci MFN, Aldrighi JM. Avaliação do estado Nutricional e do Consumo Alimentar de Mulheres no Climatério. Rev Assoc Med Bras. 2003; 49(1):91-5.

24. Mondini L, Monteiro CA. Mudanças no padrão de alimentação da população urbana brasileira (1962 - 1988). Rev Saúde Pública. 1994; 28(6): 433-9. 
25. Simopoulos AP. The importance of the ratio of omega-6/omega-3 essential fatty acids. Biomed Pharmacother. 2002; 56(8):365-79.

26. Jakovljevic J, Touillaud MS, Bondy ML, Singletary SE, Pillow PC, Chang S. Dietary intake of selected fatty acids, cholesterol and carotenoids and estrogen receptor status in premenopausal breast cancer patients. Breast Cancer Res Treat. 2002; 75(1):5-14.

27. Matsuba S, Itoh M, Joh T, Takeyama H, Dohi N, Watanabe $S$, et al. Effect of dietary linoleate/alpha-linolenate balance on experimentally induced gastric injury in rats. Prostaglandins Leukot Essent Fatty Acids. 1998; 59(5):317-23.

28. Holmes MD, Hunter DJ, Colditz GA, Stampfer MJ, Hankinson SE, Speizer FE, et al. Association of dietary intake of fat and fatty acids with risk of breast cancer. JAMA. 1999; 281(10):914-20.

29. Sugano M, Hirahara F. Polyunsaturated fatty acids in the food chain in Japan. Am J Clin Nutr. 2000; 71(1 Suppl):189s-96s.

30. Folsom AR, Demissie Z. Fish intake, marine omega3 fatty acids, and mortality in a cohort of postmenopausal women. Am J Epidemiol. 2004; 160(10):1005-10.

31. Sanders TAB. Polyunsaturated fatty acids in the food chain in Europe. Am J Clin Nutr. 2000; 71(Suppl):176s-8s.

32. Kris-Etherton PM, Taylor DS, Yu-Poth S, Moriarty K, Fishell V, Hargrove RL, et al. Polyunsaturated fatty acids in the food chain in the United States. Am J Clin Nutr. 2000; 71(1 Suppl):179s-88s.

33. Simonsen NR, Navajas JFC, Martin-Moreno JM, Strain JJ, Huttunen JK, Martin BC. Tissue Stores of Individual Monousatrated Fatty Acids And Breast Cancer: The EURAMIC Study; European Community Multicenter Study on Antioxidants, Myocardial Infarction and Breast Cancer. Am J Clin Nutr. 1998; 68(1):134-41.

34. Steinhart $H$, Rickert $R$, Winkler K. Trans fatty acids (TFA): analysis, occurrence, intake and clinical relevance. Eur J Med Res. 2003; 8(8):358-62.

35. Mensink RP, MB Katan. Effect of dietary trans fatty acids on high-density and low-density lipoprotein cholesterol levels in healthy subjects. N Engl J Med. 1990; 323(7):439-45.

36. Moreira NX, Curi, R, Padovese, R, Mancini-Filho, J. Incorporation of dietary trans monounsaturated fatty acids into tissues of Walker 256 tumorbearing rats. Braz J Med Biol Res. 2001; 34(4): 501-8.

37. Nelson GJ. Dietary fat, trans fatty, and risk of coronary heart disease. Nutr Rev. 1998; 56:250-2.
38. Bortolloto JW, Ramos GP, Souto AA. Determinação de ácidos graxos trans em margarinas por espectroscopia FTIR-ATR. In: Anais da 36 Reunião Anual da Sociedade Brasileira de Química; 2003; Poços de Caldas. Poços de Caldas: Sociedade Brasileira de Química; 2003.

39. Brasil. Ministério da Saúde. Secretaria de Vigilância Sanitária. Portaria n 521, de 17/10/97. Dispõe sobre food base. Informação nutricional complementar (521-97) CD-ROM. Brasília: Ministério da Saúde; 1997.

40. Reis C, Bortolotto J, Sousa AB, Cibeira GH, Costa S, Mottin CC, Souto AA, et al. Determinação de ácido graxo trans no tecido adiposo de uma amostra porto alegrense. In: Anais do $16^{\circ}$ Salão de Iniciação Científica; 2004; Porto Alegre. Porto Alegre: UFRGS; 2004.

41. Ip C, Banni S, Angioni E, Carta G, McGinley J, Thompson HJ, et al. Conjugated linoleic acid-enriched butter fat alters mammary gland morphogenesis and reduces cancer risk in rats. J Nutr. 1999; 129(12):2135-42.

42. Brown JM, Mclntosh MK. Conjugated linoleic acid in humans: regulation of adiposity and insulin sensitivity. J Nutr. 2003; 133(10):3041-6.

43. Vorrips LE, Brants HAM, Kardinaal AFM, Hiddink GJ, van den Brandt PA, Goldbohm RA. Intake of conjugated linoleic acid, fat, and other fatty acids in relation to postmenopausal breast cancer: the Netherlands Cohort Study on Diet and Cancer. Am J Clin Nutr. 2002; 76(4):873-82.

44. West DB, Delany JP, Camet PM, Blohm F, Truett AA, Scimeca J. Effects of conjugated linoleic acid on body fat and energy metabolism in the mouse. Am J Physiol. 1998; 275(3 Pt 2):R667-72.

45. Wu AH, Pike MC, Stram DO. Meta-analysis: dietary fat intake, serum estrogen levels, and the risk of breast cancer. J Natl Cancer Inst. 1999; 91(17): 1512.

46. Toniolo PG, Levitz M, Zeleniuch-Jacquotte $A$, Banerjee S, Koenig KL, Shore RE, et al. A prospective study of endogenous estrogens and breast cancer in postmenopausal women. J Natl Cancer Inst. 1995; 87(3):190-7.

47. Sonnenschein E, Toniolo P, Terry MB, Bruning PF, Kato I, Koeing KL, et al. Body fat distribution and obesity in pre and postmenopausal breast cancer. Int J Epidemiol. 1999; 28(6):1026-31.

48. Mannisto S, Pietinen P, Virtanen M, Kataja V, Uusitupa M. Diet and risk of breast cancer in a case control study: does the threat of disease have an influence on recall bias? J Clin Epidemiol. 1999; 52(5):429-39. 
49. Hilakivi-Clarke L, Clarke R, Onojafe I, Raygada M, Cho $E$, Lippman M. A maternal diet high in $n-6$ polyunsaturated fats alters mammary gland development, puberty onset, and breast cancer risk among female rat offspring. Proc Natl Acad Sci USA. 1997; 94(17):9372-7.

50. Sichieri R, Everhart JE, Mendonça GAS. Diet and mortality from common cancers in Brazil: an ecological study. Cad Saúde Pública. 1996; 12(1):53-9.

51. Terry P, Suzuki R, Hu FB, Wolk A. A prospective study of major dietary patterns and risk of breast cancer. Cancer Epidemiol Biomarkers Prev. 2001; 12:1281-5.

52. Graham S, Zielezny M, Marshall J, Priore R, Freudenheim J, Brasure J, et al. Diet in the epidemiology of postmenopausal breast cancer in the New York State Cohort. Am J Epidemiol. 1992; 136(11):1327-37.

53. Howe GR, Friedenreich CM, Jain M, Miller AB. A cohort study of fat intake and risk of breast cancer. J Natl Cancer Inst. 1991; 83(5):336-40.
54. Kushi LH, Sellers TA, Potter JD, Nelson CL, Munger RG, Kaye SA, et al. Dietary fat and postmenopausal breast cancer. J Natl Cancer Inst. 1992; 84(14): 1092-9.

55. Howe GR, Hirohata T, Hislop TG, Iscovich JM, Yuan JM, Katsouyanni K, et al. Dietary factors and risk of breast cancer: combined analysis of 12 case control studies. J Natl Cancer Inst. 1990; 82(7): 561-9.

56. Byers T, Nestle M, McTiernan A. Guidelines on Nutrition and Physical Activity for Cancer Prevention: recommendations for individuals [Internet]. Atlanta: American Cancer Society; 2005. Available from: http://www.cancer.org/downloads/ STT/CPED2005v5PWSecured.pdf

Recebido em: 3/2/2004

Versão final reapresentada em: 31/5/2005

Aprovado em: 21/6/2005 